\title{
Spatially variant PSF modeling and image deblurring
}

\author{
Éric Thiébaut ${ }^{\mathrm{a}}$, Loïc Denis ${ }^{\mathrm{b}}$, Ferréol Soulez ${ }^{\mathrm{a}, \mathrm{c}}$, and Rahul Mourya ${ }^{\mathrm{b}}$ \\ ${ }^{\mathrm{a}}$ Univ. Lyon, Univ. Lyon 1, ENS de Lyon, CNRS, Centre de Recherche Astrophysique de Lyon UMR5574, F-69230, \\ Saint-Genis-Laval, France \\ ${ }^{\mathrm{b}}$ Univ Lyon, UJM-Saint-Étienne, CNRS, Institut d'Optique Graduate School, Laboratoire Hubert Curien UMR 5516, \\ F-42023, Saint-Étienne, France \\ ${ }^{\mathrm{c} B i o m e d i c a l ~ I m a g i n g ~ G r o u p, ~ E ́ c o l e ~ P o l y t e c h n i q u e ~ F e ́ d e ́ r a l e ~ d e ~ L a u s a n n e ~(E P F L), ~ C H-1015 ~ L a u s a n n e, ~ S w i t z e r l a n d . ~}$
}

\begin{abstract}
Most current imaging instruments have a spatially variant point spread function (PSF). An optimal exploitation of these instruments requires to account for this non-stationarity. We review existing models of spatially variant PSF with an emphasis on those which are not only accurate but also fast because getting rid of non-stationary blur can only be done by iterative methods.
\end{abstract}

Keywords: Variable PSF; image processing; fast and accurate non-stationary blur approximation

\section{INTRODUCTION}

High angular resolution images are now routinely produced by astronomical telescopes thanks to adaptive optics systems. Unless considering very narrow fields of view, these nearly diffraction-limited images are however affected by point spread functions (PSF) which are spatially variable. For instance, Fig. 1 shows an image of the Galactic Center obtained with PUEO adaptive optics system at CFHT. ${ }^{1}$ Clearly, the PSF near the guiding star has a high Strehl ratio while more distant regions appear to be more blurred. Non-stationary PSF is not restricted to high angular resolution imaging, it is a general rule rather than an exception for many current astronomical instruments, in particular, large integral field spectrographs like MUSE. ${ }^{2}$ In order to retrieve images at the best spatial and spectral resolution from astronomical data, deblurring algorithms that work for a spatially variant PSF must be available. Compared to the simpler deconvolution problem, dealing with a variable PSF for deblurring astronomical images (possibly multi-spectral) however has several specific issues. First, a good approximation of a shift-variant blur is needed to correctly estimate physical parameters such as the photometry. Second, getting rid of a spatially variant blur is necessarily an iterative process, so applying the model of the blur (and its adjoint) should be a fast operation. Third, practical means to calibrate a shift variant blur must be found. In this contribution, we review existing blur models with a particular emphasis on the models which are the most suitable for astronomy and for being used in restoration methods.

\subsection{Image Formation Model}

For an incoherent object, the general model for the distribution of light in the image plane of an imaging instrument takes the form of a Fredholm equation of the first kind:

$$
g(r)=\int h(r, s) f(s) \mathrm{d} s
$$

where $r$ is the position in the image, the kernel $h(r, s)$ is the point spread function (PSF) and $f(s)$ is the object brightness distribution at position $s$. To simplify the equations, $r$ and $s$ must be expressed in the same system of coordinates. In the following, we assume that they are both angular directions. Introducing the centered PSF $\kappa(q, s) \equiv h(q+s, s)$ which is the observed distribution for a point source at an angular offset $q$ with respect to the position $s$ of the source, the model in Eq. (1) becomes:

$$
g(r)=\int \kappa(r-s, s) f(s) \mathrm{d} s .
$$

E-mail: eric.thiebaut@univ-lyon1.fr

Adaptive Optics Systems V, edited by Enrico Marchetti, Laird M. Close, Jean-Pierre Véran, Proc. of SPIE Vol. 9909, 99097N (C) 2016 SPIE · CCC code: 0277-786X/16/\$18 · doi: 10.1117/12.2233647 


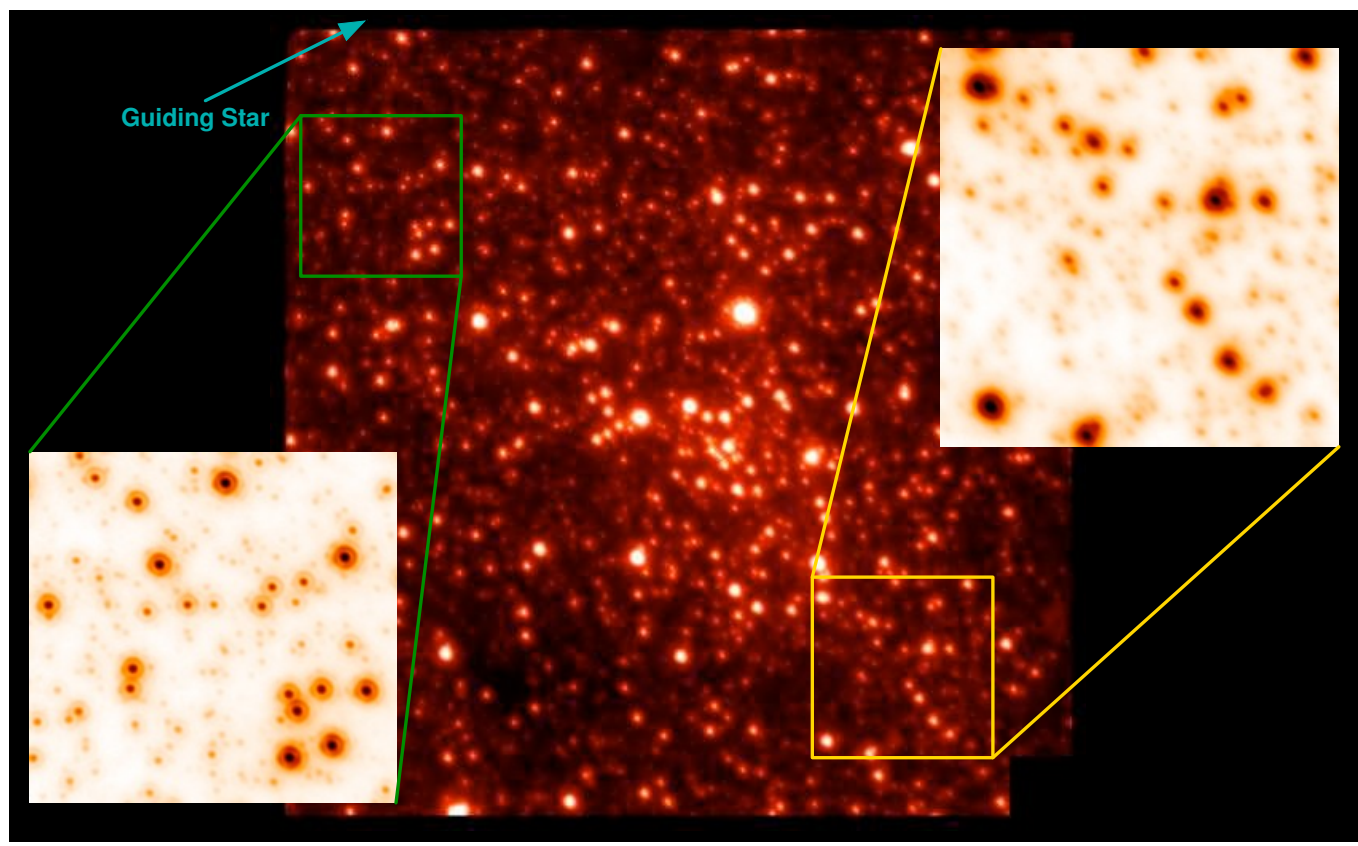

Figure 1. Images of the Galactic center with PUEO adaptive optics system. The green box shows a region which is close to the guide star and hence has a nearly perfect diffraction limited PSF while the region in the yellow box is more distant from the guiding star and is affected by a degraded PSF. Credits: Flicker \& Rigaut, $2004^{1}$

\subsection{PSF Properties}

The PSF $h(r, s)$ (resp. $\kappa(q, s)$ ) can be seen as the probability density function of the position $r$ (resp. of the offset $q$ ) of a detected photon conditioned to the position $s$ of the source. ${ }^{3}$ It is therefore expected that the PSF be nonnegative and normalized in the sense that:

$$
\begin{array}{rlll}
h(r, s) \geq 0 & (\forall r, \forall s) & \kappa(q, s) \geq 0 & (\forall q, \forall s), \\
\int h(r, s) \mathrm{d} r=1 & (\forall s) & \int \kappa(q, s) \mathrm{d} q=1 & (\forall s) .
\end{array}
$$

Preserving these properties may be of utmost importance, for instance to accurately recover the photometry of sources in the restored image. As an illustration, Fig. 2 shows the effect of the blurring by a variable PSF on an image of the galaxy M64, and the deblurred images assuming a shift invariant PSF and using a good approximation of the actual blur. Clearly using an improper blur model for image restoration yields much worse results.

\subsection{Discretization}

In reality, the observed distribution $g(r)$ is sampled by the pixels of the detector and perturbed by noise. The measurement given by the $i$-th pixel of the detector then writes:

$$
d_{i}=g\left(r_{i}\right)+n_{i}=\int h\left(r_{i}, s\right) f(s) \mathrm{d} s+n_{i}=\int \kappa\left(r_{i}-s, s\right) f(s) \mathrm{d} s+n_{i},
$$

where $r_{i}$ is the angular direction seen by the $i$-th pixel and $n_{i}$ is a stochastic term due to the noise. Note that the PSF $h(r, s)$ and the centered PSF $\kappa(q, s)$ must account for the integration by the pixels for the above relations to hold.

Deblurring involves the restoration of a crisp image which is an approximation of $f(s)$ the brightness distribution of the object. For practical reasons, a discrete representation of the sought image has to be chosen. For instance, using Riemann sum to approximate the integral yields:

$$
g\left(r_{i}\right)=\int h\left(r_{i}, s\right) f(s) \mathrm{d} s \approx \sum_{j=1}^{N} h\left(r_{i}, s_{j}\right) f\left(s_{j}\right) \Delta s_{j},
$$



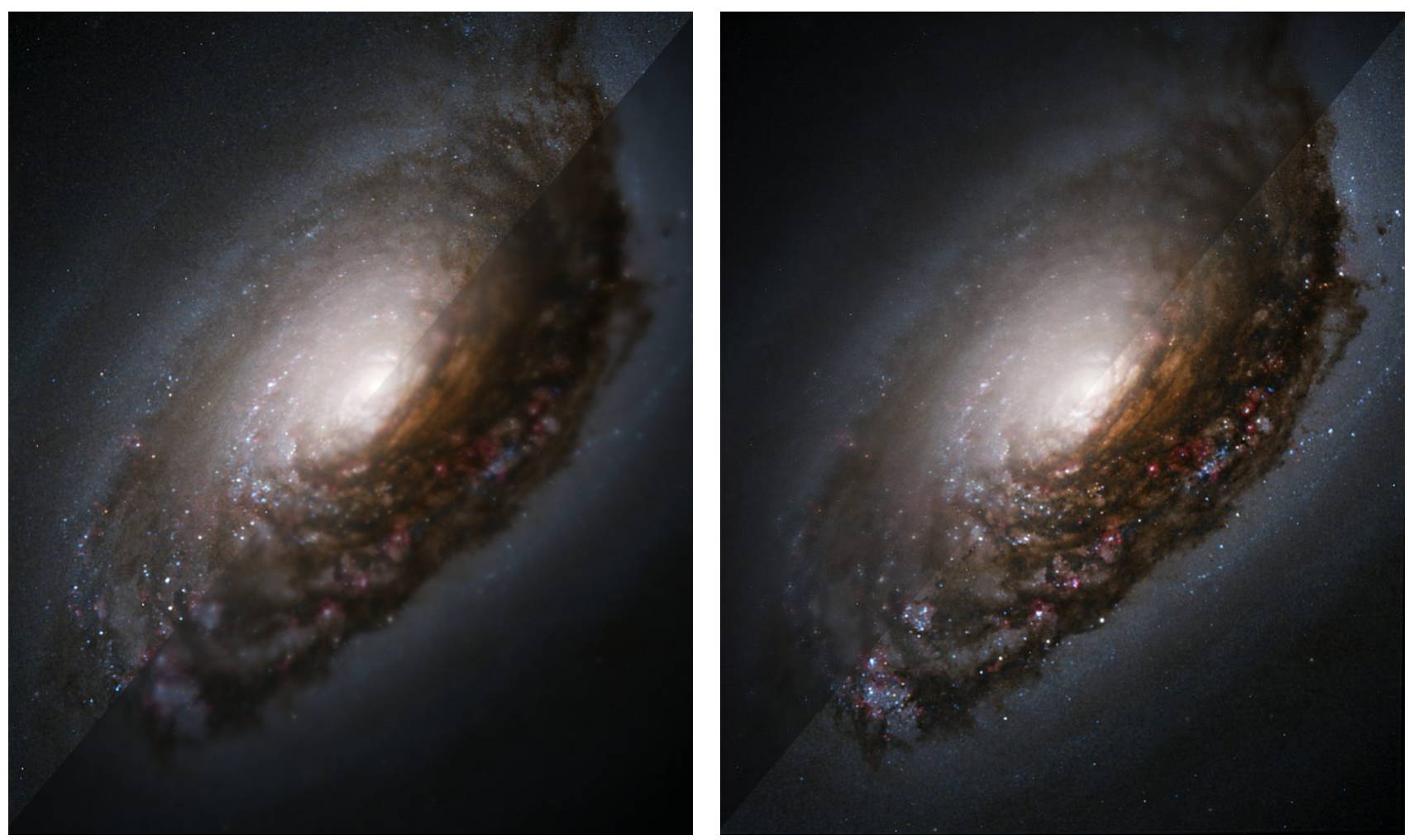

Figure 2. Images of the galaxy M64. Left: a montage showing the galaxy at full resolution / blurred by a shift-variable PSF. Right: a montage showing the restored images assuming a shift invariant PSF / with a shift variant model of the blur. Credits: NASA and the Hubble Heritage Team (AURA/STScI) for the original image of M64 (http://hubblesite. org/gallery/album/galaxy/pr2004004a/).

with $\left\{s_{j}\right\}_{j=1, \ldots, N}$ a list of sampled source positions and $\Delta s_{j}$ the effective volume of integration associated to $s_{j}$. Clearly, $\left\{x_{j} \equiv f\left(s_{j}\right)\right\}_{j=1, \ldots, N}$ are the unknowns of the image deblurring problem. In general, the list of sampled positions forms a rectangular grid and $x=\left(x_{1}, \ldots, x_{N}\right)^{\mathrm{t}}$ is a simple pixelized image. Using matrix notation, the direct model of the data writes:

$$
d=H x+n,
$$

where $d=\left(d_{1}, \ldots, d_{M}\right)^{\mathrm{t}}$ represents the data (that is the values measured by the $M$ pixels of the detector) and $H \in \mathbb{R}^{M \times N}$ is a discrete linear operator whose coefficients are given by:

$$
H_{i, j}=h\left(r_{i}, s_{j}\right) \Delta s_{j}=\kappa\left(r_{i}-s_{j}, s_{j}\right) \Delta s_{j} .
$$

In what follows, we make use of the discrete centered PSF $K \in \mathbb{R}^{L \times N}$ defined by:

$$
K_{\ell, j}=\kappa\left(q_{\ell}, s_{j}\right) \Delta s_{j}
$$

for some list $\left\{q_{\ell}\right\}_{\ell=1, \ldots, L}$ of sampled offsets. Using the discrete centered PSF introduces some technical complications to write the direct model of the data:

$$
d_{i}=\sum_{j=1}^{N} K_{\ell(i, j), j} x_{j}+n_{i},
$$

where the mapping $\ell(i, j)$ yields the index such that $q_{\ell(i, j)}=r_{i}-s_{j}$. This relation implies that the two sampling lists, $\left\{r_{i}\right\}_{i=1, \ldots, M}$ and $\left\{s_{j}\right\}_{j=1, \ldots, N}$, correspond to the same rectangular grid with even spacing. 


\subsection{Image Deblurring}

Given the data $d=\left(d_{1}, \ldots, d_{M}\right)^{\mathrm{t}}$ and their model, e.g. Eq. (5), image deblurring amounts to finding a good approximation of the image parameters $x$. This is an inverse problem* which is customarily recast as an optimization problem: ${ }^{4-6}$

$$
\widehat{x}=\underset{x \geq 0}{\arg \min }\{\mathcal{F}(x)=\mathcal{L}(x)+\mu \mathcal{R}(x)\},
$$

where minimizing $\mathcal{L}(x)$ enforces agreement of the model with the data while minimizing $\mathcal{R}(x)$ imposes that the solution be somewhat regular. The so-called hyper-parameter $\mu \geq 0$ is used to tune the trade-off between over-fitting the data (and thus amplifying the noise) and over-regularizing the solution. The notation $x \geq 0$ in Eq. (9) indicates that we want to constrain the solution to be nonnegative everywhere for we known it is a brightness distribution.

For an optimal exploitation of the measurements, the data fidelity penalty $\mathcal{L}(x)$ should be derived from the co-log-likelihood of the data. Assuming Gaussian noise leads to:

$$
\mathcal{L}(x)=\frac{1}{2}\|H x-d\|_{W}^{2},
$$

with $\|u\|_{W}^{2} \stackrel{\text { def }}{=} u^{\mathrm{t}} W u$ the weighted squared Euclidean norm and $W$ a positive semi-definite linear operator implementing statistical weighting of the model errors. If there are no invalid or missing data, $W$ is the inverse of $\operatorname{Cov}(n \mid x)$ the covariance matrix of the noise conditioned to the knowledge of the parameters $x$. For astronomical images, the true noise distribution is not Gaussian, at least because of the Poissonian distribution of the detected photons. Nevertheless, assuming a non-homogeneous independent Gaussian noise usually provides a good approximation and practical means to derive the noise variance from the data have been proposed. ${ }^{7,8}$

The penalty $\mathcal{R}(x)$ is a regularization term needed to cope with the ill-conditioned nature of the deblurring problem and to avoid, among others, artifacts due to noise amplification. In practice, $\mathcal{R}(x)$ should favor a regular or simple solution. That is to say, smooth or expressed by very few significant values. Simple considerations ${ }^{6}$ lead to design a regularization $\mathcal{R}(x)$ which is invariant ${ }^{\dagger}$ by simple geometrical transforms of the sought object $x$. A quadratic regularization combined with the data fidelity term defined by Eq. (10) yields a closed form solution which must nevertheless be computed by means of iterative methods and which, owing to the high contrast of astronomical images, displays spurious ripples. A non-quadratic regularization can help avoiding these artifacts. Total variation ${ }^{9}$ (TV) has emerged as the de facto standard in image restoration but it yields piecewise flat images that may not be appropriate for astronomical sources and edge preserving regularizations ${ }^{7,10}$ are probably more suitable for resolved astronomical objects. For unresolved sources (e.g. the stars in a cluster), separable sparsity can be favored by using the $\ell_{1}$ norm of the sought image. ${ }^{11}$ Finally, it is possible to design a regularization $\mathcal{R}(x)$ so as to account for a mixture of unresolved sources on top of a rather smooth background, ${ }^{12}$ this however requires to decompose the unknown parameters in two subsets: a smooth image and a map of point-like sources.

Once chosen the terms of the penalty in Eq. (9), numerical iterative methods are needed to find the solution $\widehat{x}$ which is unique provided $\mathcal{F}(x)$ be strictly convex. If $\mathcal{F}(x)$ is differentiable with respect to $x$, limited memory implementations of constrained quasi-Newton methods ${ }^{13}$ are probably the easiest methods to use. For nondifferentiable penalties as TV or the $\ell_{1}$ norm, augmented Lagrangian methods ${ }^{14}$ or alternating directions of multipliers methods ${ }^{15,16}$ (ADMM) are very effective and quite easy to implement.

\section{FAST SHIFT-VARIANT BLUR MODEL}

The general image formation model with a shift-variant PSF is impractical as it requires the storage of $L N$ PSF coefficients and applying the model takes $2 L N$ numerical operations. For a $N=1000 \times 1000$ pixel crisp image and centered PSFs of size $L=100 \times 100$, operator $K$ requires $\sim 40 \mathrm{~Gb}$ of storage with 32 -bit floating-point values

\footnotetext{
*by opposition to the problem of deriving a proper model of the data which is termed as the direct problem

${ }^{\dagger}$ except perhaps by a factor which can be compensated by adjusting $\mu$
} 


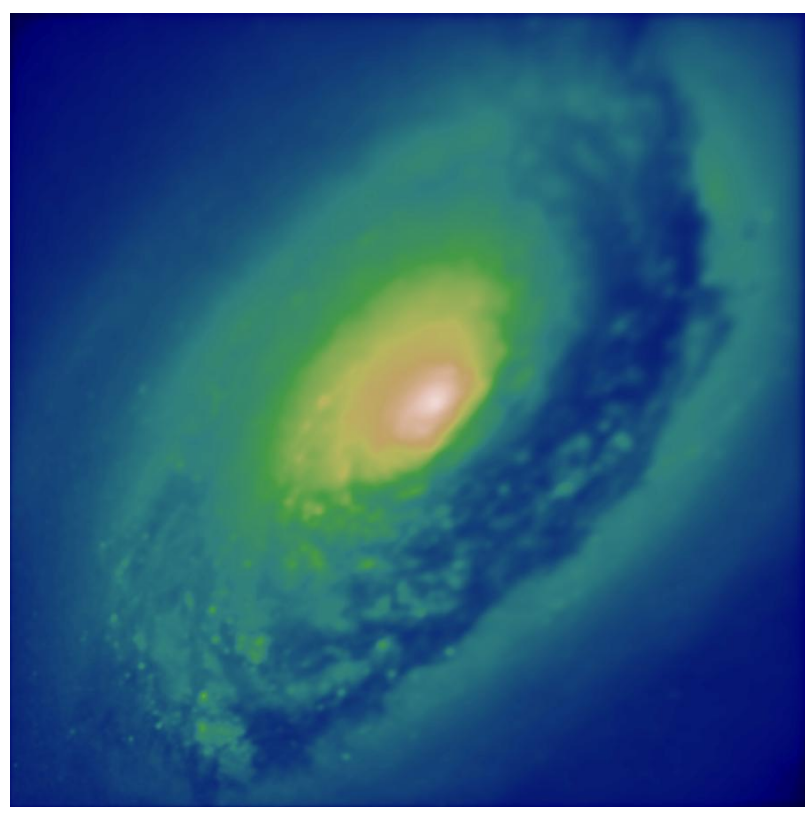

Figure 3. The galaxy M64 blurred by a variable PSF. The optical center is located near the lower left corner where the blur is less severe.

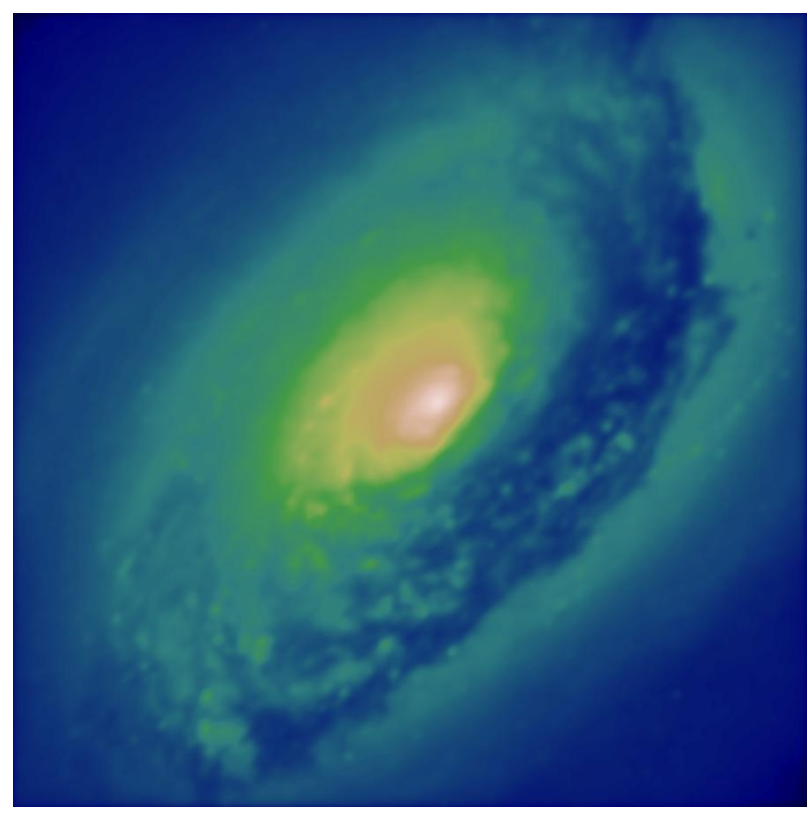

Figure 4. The galaxy M64 blurred by a shift-invariant PSF. As a consequence, the blur is the same everywhere.

and takes $\sim 20 \times 10^{9}$ floating-point operations to be applied. Comparatively, with a shift invariant PSF, blurring amounts to a simple discrete convolution which can be computed by means of fast Fourier transforms (FFT) in $O(N \log N)$ floating-point operations. Because the deblurring problem (9) can only be solved by iterative methods, it is important to implement fast computation of the blurring while preserving a good approximation of the real blur.

In order to compare the different approximations of a variable blur, we have simulated the effect of shiftvariant blur of NACO (SWAN) ${ }^{17}$ on a crisp image of M64 galaxy from Hubble Space Telescope (source: http: //hubblesite.org/gallery/album/galaxy/pr2004004a/, Credits: NASA and The Hubble Heritage Team (AURA/STScI)). This image has structures at many different spatial scales and is thus useful to illustrate the difference between blur models. Fig. 2) shows the original color image aside with a blurred version and reconstructions with different blur models. Figure 3 shows the brightness distribution of the galaxy as if observed by the HST assuming the optical center is located near the lower left corner where the blur is less severe. Subsequent figures will show the blurred images under various approximations using the same colormap.

\subsection{Shift-invariant Approximation}

The most obvious and simple approach consists in assuming that the PSF is stationary (shift-invariant) and thus take $h(r, s)=\kappa(r-s)$ or, equivalently, $\kappa(q, s)=\kappa(q)$ where $\kappa(q)$ is, for instance, the PSF for a given direction $s$ or the mean PSF across the field of view. This amounts to neglecting the PSF variations and only provides a good approximation in the so-called isoplanatic domain. Under this approximation, the image formation model becomes a simple convolution:

$$
g(r)=\int \kappa(r-s) f(s) \mathrm{d} s=(\kappa \circledast f)(r),
$$

where $\circledast$ denotes angular convolution. The amount of memory is only that of a single PSF and, as said before, the computational burden is that of a full size discrete convolution that is $O(N \log N)$.

Figure 4 displays the image of the galaxy M64 blurred with a shift invariant PSF (assumed to be the one at the center of the field of view), compared to the effects of the actual PSF ( $c f$. Fig. 3), the approximation is only good in the center of the image (the blur is too strong in the lower left part and insufficient in the upper right part). 


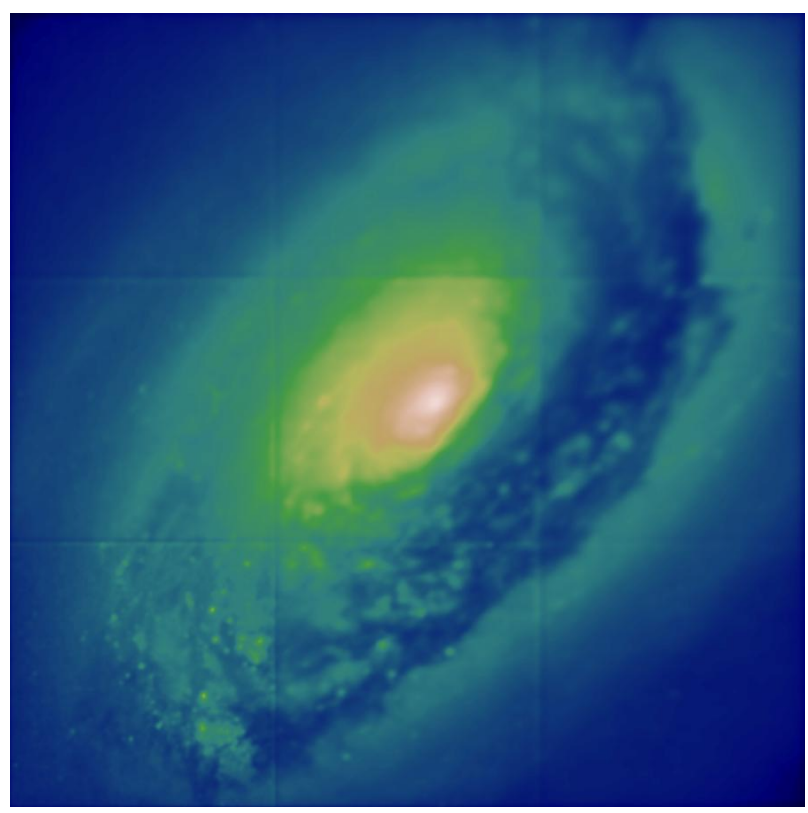

Figure 5. The galaxy M64 blurred by a PSF which is shift invariant by block. The artifacts at the edges of the blocks are obvious.

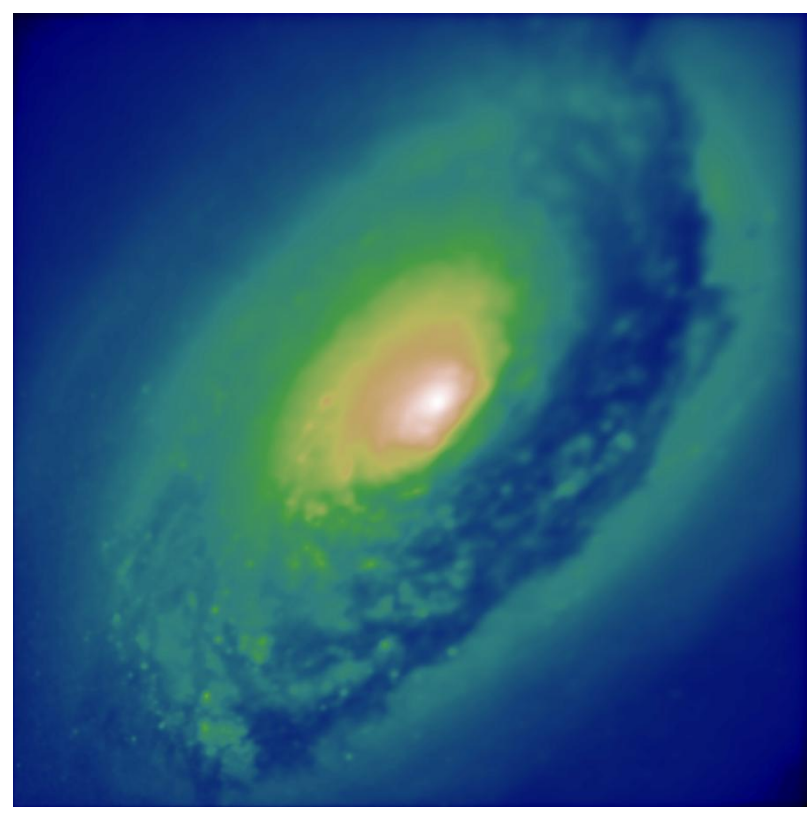

Figure 6 . The galaxy M64 blurred by a variable PSF following Nagy \& O'Leary ${ }^{20}$ with a grid of $4 \times 4$ PSF.

\subsection{Piecewise Approximation}

In order to exploit fast convolution by means of FFT, many authors (e.g. Ciliegi et al. ${ }^{18}$ for a recent example) were content to carve the field of view in small regions where they assumed the PSF to be stationary. Each region is blurred by its respective PSF and the blurred regions are assembled to form a large image. Given $\left\{c_{k}\right\}_{k=1, \ldots, P}$ the positions at the center of the $P$ regions, such an approximation of the blur by a variable PSF can be formally expressed as:

$$
g(r) \approx \sum_{k=1}^{K} \iota_{k}(r) \int \kappa\left(r-s, c_{k}\right) f(s) \mathrm{d} s=\sum_{k=1}^{K} \iota_{k}(r)\left(\kappa_{k} \circledast f\right)(r),
$$

where $\iota_{k}(r) \in\{0,1\}$ is the indicative function of the $k$-th region (which is equal to 1 inside the region and 0 elsewhere) and $\kappa_{k}(q) \stackrel{\text { def }}{=} \kappa\left(q, c_{k}\right)$ is the PSF for the $k$-th region. From the above expression it is immediately deduced that the computational cost is $O(N \log (N / P))$ floating point operations if the sizes of PSFs are small compared to the sizes of the regions. Note that Eq. (12) amounts to assuming that:

$$
h(r, s) \approx \sum_{k=1}^{K} \iota_{k}(r) \kappa\left(r-s, c_{k}\right)=\sum_{k=1}^{K} \iota_{k}(r) \kappa_{k}(r-s),
$$

which is a piecewise constant approximation of the variable PSF. There is no simple expression for the equivalent centered PSF, $\kappa(q, s)$, however see Denis et al. ${ }^{19}$ As shown by the artifacts at the boundaries of the regions shown in Fig. 5, the main issue of this rather crude approximation is the lack of continuity in the direct model of the blur.

\subsection{Improvement by Interpolating the Piecewise Approximation}

A progressive blending of the regions blurred by different PSFs provides an ad-hoc method to smooth out the boundary artifacts. This is essentially the model of a shift variant blur proposed by Nagy \& O'Leary ${ }^{20}$ which writes:

$$
g(r) \approx \sum_{k=1}^{P} \phi_{k}(r) \int \kappa\left(r-s, c_{k}\right) f(s) \mathrm{d} s=\sum_{k=1}^{K} \phi_{k}(r)\left(\kappa_{k} \circledast f\right)(r),
$$


where $\phi_{k}(r) \in[0,1]$ is a chosen continuous interpolation function. For instance, taking $\phi_{k}(r)=\Lambda\left(r-c_{k}\right)$ with $\Lambda(q)$ the separable triangle function yields bilinear interpolation. The corresponding PSF is given by:

$$
h(r, s) \approx \sum_{k=1}^{K} \phi_{k}(r) \kappa\left(r-s, c_{k}\right)=\sum_{k=1}^{K} \phi_{k}(r) \kappa_{k}(r-s) .
$$

The formal similarity with Eq. (12) and (13) is apparent: $\iota_{k}(r)$ has just been replaced by $\phi_{k}(r)$. If the interpolating functions have a finite support, not all PSFs have an incidence on a given image position $r$ and the approximation reduces to:

$$
h(r, s) \approx \sum_{k \in \mathbb{S}(r)} \phi_{k}(r) \kappa_{k}(r-s),
$$

where $\mathbb{S}(r)$ is the set of indexes $k$ of the PSFs which have to taken into account for computing the blur at position $r$. To benefit from the blending, the blurred regions have to overlap. In $2 \mathrm{D}$, each position typically requires to compute a convolution by $2 \times 2 \mathrm{PSFs}$, hence Card $(\mathbb{S}(r))=4$ and the computational burden is $O(4 N \log (N / P))$ floating point operations. Figure 6 shows that this model of blur yields acceptable results, at least much better than the piecewise approximation. This model is however not as good as the two which follow.

\subsection{Modal PSF Approximation}

It can be observed that the centered PSF $\kappa(q, s)$ has less variations in $s$ than the non-centered PSF $h(r, s)(c f$. Table 1 in Denis et al. $\left.{ }^{19}\right)$. As proposed by Flicker \& Rigaut, ${ }^{21}$ it seems then natural to decompose the PSF in a limited number of modes to form the following approximation:

$$
\kappa(q, s) \approx \sum_{k=1}^{P} p_{k}(q) w_{k}(s)
$$

where $\left\{p_{k}(q)\right\}_{k=1, \ldots, P}$ and $\left\{w_{k}(s)\right\}_{k=1, \ldots, P}$ are the so-called left and right modes of the decomposition. With such an approximation, the image formation model becomes:

$$
g(r) \approx \int\left(\sum_{k=1}^{P} p_{k}(r-s) w_{k}(s)\right) f(s) \mathrm{d} s=\sum_{k=1}^{P} \int p_{k}(r-s)\left(w_{k} \otimes f\right)(s) \mathrm{d} s=\sum_{k=1}^{P}\left(p_{k} \circledast\left(w_{k} \otimes f\right)\right)(r),
$$

where $\otimes$ denote componentwise multiplication, i.e. $\left(w_{k} \otimes f\right)(s)=w_{k}(s) f(s)$, and $\circledast$ denotes angular convolution. The final expression in Eq. (18) makes clear that this approximation amounts to weighting the incoming distribution $f(s)$ by the right modes $w_{k}(s)$ before convolving with the left modes $p_{k}(s)$.

In practice, the singular value decomposition ${ }^{22,23}$ (SVD) yields the left and right PSF modes. The SVD of the discrete centered PSF $K$ is given by:

$$
K=U \Sigma V^{\mathrm{t}}=\sum_{k=1}^{\min (L, N)} \sigma_{k} u_{k} v_{k}^{\mathrm{t}}
$$

where $U$ and $V$ are unitary matrices whose respective columns (denoted as $u_{k}$ and $v_{k}$ above) are the so-called left and right singular vectors while $\Sigma$ is a, possibly rectangular, diagonal matrix whose diagonal coefficients (denoted as $\sigma_{k}$ above) are the so-called singular values. The singular values are nonnegative and, by convention, sorted in descending order; furthermore $\sigma_{k}=0$ for any $k>\operatorname{rank}(K) \leq \min (L, N)$. Eckard-Young-Mirsky theorem ${ }^{23,24}$ demonstrates that the truncated singular value decomposition (TSVD):

$$
K \approx K^{(P)}=\sum_{k=1}^{P} \sigma_{k} u_{k} v_{k}^{\mathrm{t}}
$$

with $P \leq \min (L, N)$ is the best approximation of rank $P$ of the matrix $K$ in the least squares sense. The approximation is unique if $\sigma_{P+1}<\sigma_{P}$ and the approximation is exact if $P \geq \operatorname{rank}(K)$. 
Introducing the decomposition of Eq (17), the discrete centered PSF defined in Eq. (7) writes:

$$
K_{\ell, j}=\kappa\left(q_{\ell}, s_{j}\right) \Delta s_{j} \approx \sum_{k=1}^{P} p_{k}\left(q_{\ell}\right) w_{k}\left(s_{j}\right) \Delta s_{j}=K_{\ell, j}^{(P)},
$$

where the last equality holds if the sampled PSF modes are chosen as follows:

$$
\begin{aligned}
p_{k}\left(q_{\ell}\right) & =\left(\sigma_{k} / \alpha_{k}\right) U_{\ell, k}, \\
w_{k}\left(s_{j}\right) & =\left(\alpha_{k} / \Delta s_{j}\right) V_{j, k},
\end{aligned}
$$

where the $\alpha_{k}$ are arbitray factors (provided that $\alpha_{k} \neq 0, \forall k$ ). One issue is the size of the matrix to which apply the SVD, this may be alleviated by subsampling the PSF $\kappa(q, s)$ with respect to the input position $s$ (i.e. assuming that is varies slowly with $s$ ). Another more critical issue is that if the PSF varies significantly across the field of view many modes will be needed to correctly mimic the PSF. Finally, the PSF modes have no physical meanings and it is likely that properties like nonnegativity or flux conservation will not hold everywhere for the truncated mode approximation. The computational burden of applying the modal PSF approximation is that of $P$ full size discrete convolutions hence $O(P N \log N)$ operations.

\subsection{PSF Interpolation}

Let us assume that the centered PSF $\kappa(q, s)$ is known for a set of $P$ calibration sources at positions $\left\{c_{k}\right\}_{k=1, \ldots, P}$, then, considering the continuity of the variation of the centered PSF with respect to the source position $s$, we have proposed to approximate the PSF at a given position by interpolating the nearby calibrated PSF: ${ }^{25}$

$$
\kappa(q, s) \approx \sum_{k \in \mathbb{K}(s)} \varphi_{k}(s) \kappa\left(q, c_{k}\right),
$$

where $\mathbb{K}(s)$ denotes the set of indexes $k$ of the calibration PSFs which have to taken into account to interpolate the blur at position $s$ and $\left\{\varphi_{k}(s)\right\}_{k=1, \ldots, P}$ are the chosen interpolating functions. ${ }^{26}$ Usually, although this is not required, the interpolating functions are shifted versions of the same function: $\varphi_{k}(s)=\varphi\left(s-c_{k}\right)$. The comparison of Eq. (22) with Eq. (17) readily shows that PSF interpolation corresponds to a modal decomposition with a specific choice for the left and right modes:

$$
\begin{aligned}
& p_{k}(q)=\kappa\left(q, c_{k}\right), \\
& w_{k}(s)=\varphi\left(s-c_{k}\right),
\end{aligned}
$$

up to some arbitrary factors as for Eqs. (21a)-(21b). Based on computational considerations Hirsh et al. ${ }^{27}$ have proposed an expression of the spatially variant blur which amounts to our model.

In spite of the close formal resemblance of the modal decomposition provided by TSVD and the approximation by PSF interpolation, they have different practical properties. For the same number $P$ of modes and of calibrated PSF, TSVD is guaranteed to provide the best approximation (in the least squares sense) of the true PSF but it does not impose any constraints on the modes. The PSF interpolation approximation can, quite easily, preserve properties such as nonnegativity and normalization provided they hold for the calibrated PSF $\kappa\left(q, c_{k}\right)$. We have shown that other properties such as symmetries or invariances can also be preserved by interpolating the PSF. ${ }^{19}$ Furthermore, the interpolating model can be greatly improved by fitting the calibration PSFs and the interpolation weights. ${ }^{19,25}$ Although it impacts the computational burden, refining the grid of calibrated PSF's is another way to improve the accuracy as the interpolated PSF model converges to the actual shift variant PSF as the calibration PSFs become closer and closer. The model of Nagy \& O'Leary ${ }^{20}$ does not have such property neither it preserves the flux. Finally, another advantage of the interpolating model is that its computational time remains modest. In the limit of recentered PSF with small support, applying the shift variant blur operator $H$ (or its adjoint $H^{\mathrm{t}}$ ) takes 4 times the time of a simple convolution. 


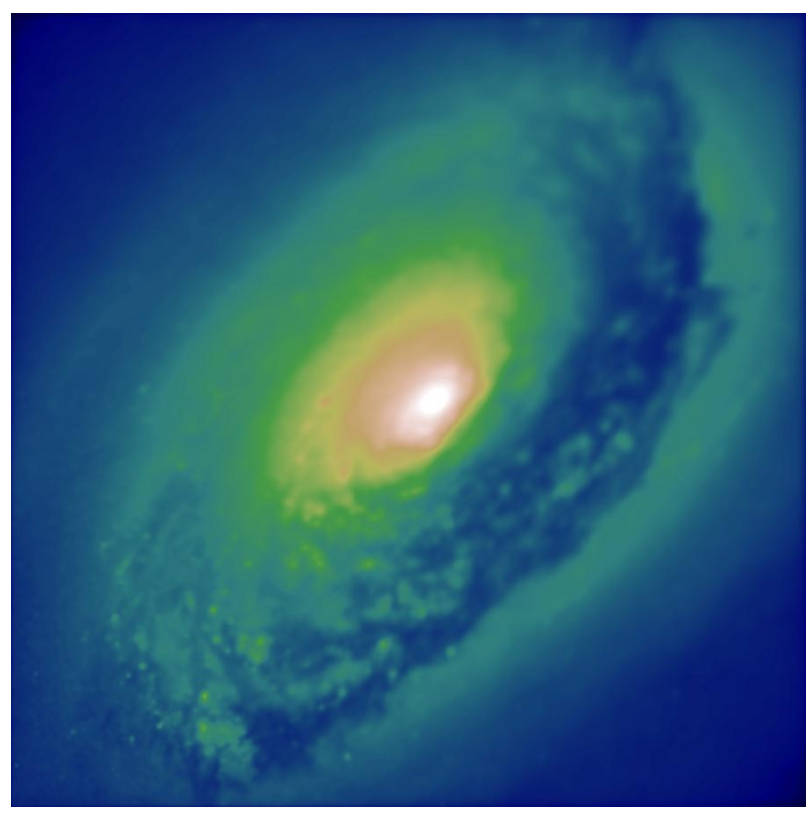

Figure 7. The galaxy M64 blurred by Flicker \& Rigaut ${ }^{21}$ modal PSF with 16 modes.

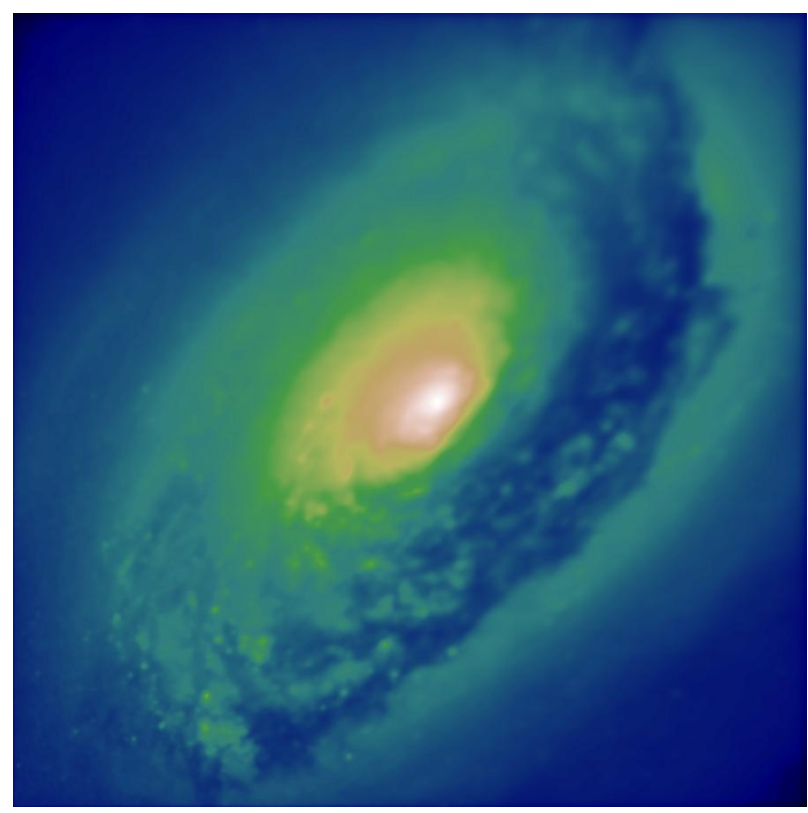

Figure 8. The galaxy M64 blurred by the proposed variable PSF model interpolated on a grid of $4 \times 4$ calibration PSF.

\section{DISCUSSION AND PERSPECTIVES}

We have reviewed a number of proposed models of shift variant PSFs and shown that the interpolating model ${ }^{19,25,27}$ yields a good approximation which preserves properties of the PSF while being also very fast to apply. Maalouf et al. ${ }^{28}$ have proposed to deblur an image affected by a shift-variant PSF by interpolating between the results of deconvolving with different PSFs. We however expect that optimal results can be obtained by using the proposed shift variant PSF in an iterative method for solving inverse problems. Such algorithms require to apply the operator $H$ (and its adjoint $H^{\mathrm{t}}$ ) many times and it is important that the blur model provides not only good but also fast approximation.

Perhaps the most important next step is to develop means to calibrate shift variant PSF especially when it depends on the observing conditions as it is the case in astronomy and microscopy. In that context, selfcalibration methods which yield at the same time the deblurred image an the instrumental response are the most appealing ones even though they pose additional difficulties. There have been some very encouraging results on both empirical or synthetic images ${ }^{29}$ and with physical models of the PSF accounting for Fresnel propagation in the instrument. ${ }^{30}$

\section{ACKNOWLEDGMENTS}

The research leading to these results has received funding from the French Agence Nationale pour la Recherche (ANR) under grant agreement number ANR DEFI 09-EMER-008-01 (MiTiV project http://mitiv . univ-lyon1. fr).

\section{REFERENCES}

[1] Flicker, R. and Rigaut, F., "New method for deconvolving adaptive optics images," in [Workshop on Adaptive Optics PSF Reconstruction], Herzberg Institute of Astrophysics, National Research Council of Canada (2004).

[2] Soulez, F., Thiébaut, É., and Denis, L., "Restoration of hyperspectral astronomical data with spectrally varying blur," New Astronomy Review 59, 403-416 (2013).

[3] Richardson, W. H., "Bayesian-based iterative method of image restauration," J. Opt. Soc. America 62(1), 55-59 (1972). 
[4] Titterington, D. M., "General structure of regularization procedures in image reconstruction," Astron. Astrophys. 144, 381-387 (1985).

[5] Thiébaut, É., "Introduction to image reconstruction and inverse problems," in [Optics in Astrophysics], Foy, R. and Foy, F.-C., eds., NATO ASI 198, 397-422, Springer, Amsterdam (2005).

[6] Thiébaut, É., "Principles of image reconstruction in interferometry," EAS Publications Series 59, $157-187$ (2013).

[7] Mugnier, L. M., Fusco, T., and Conan, J.-M., "MISTRAL: a myopic edge-preserving image restoration method, with application to astronomical adaptive-optics-corrected long-exposure images," J. Opt. Soc. America A 21, 1841-1854 (Oct. 2004).

[8] Foi, A., Trimeche, M., Katkovnik, V., and Egiazarian, K., "Practical poissonian-gaussian noise modeling and fitting for single-image raw-data," IEEE Transactions on Image Processing 17, 1737-1754 (2008).

[9] Rudin, L., Osher, S., and Fatemi, E., "Nonlinear total variation based noise removal algorithms," Physica D 60, 259-268 (1992).

[10] Charbonnier, P., Blanc-Féraud, L., Aubert, G., and Barlaud, M., "Deterministic edge-preserving regularization in computed imaging," IEEE Trans. Image Process. 6, 298-311 (feb 1997).

[11] Donoho, D., "For most large underdetermined systems of linear equations, the minimal ell-1 norm near-solution approximates the sparsest near-solution," Communications on Pure and Applied Mathematics 59(7), 907-934 (2006).

[12] Giovannelli, J.-F. and Coulais, A., "Positive deconvolution for superimposed extended source and point sources," Astron. Astrophys. 439, 401-412 (Aug. 2005).

[13] Thiébaut, É., "Optimization issues in blind deconvolution algorithms," in [Astronomical Data Analysis II], Starck, J.-L. and Murtagh, F. D., eds., 4847, 174-183, SPIE (2002).

[14] Bertsekas, D. P., [Nonlinear programming], Athena Scientific (1999).

[15] Gabay, D. and Mercier, B., "A dual algorithm for the solution of nonlinear variational problems via finite element approximation," Computers $\&$ Mathematics with Applications 2(1), 17-40 (1976).

[16] Boyd, S., Parikh, N., Chu, E., Peleato, B., and Eckstein, J., "Distributed optimization and statistical learning via the alternating direction method of multipliers," Foundations and Trends in Machine Learning 3, 1-122 (2010).

[17] Cresci, G., Davies, R., Baker, A., and Lehnert, M., "Accounting for the anisoplanatic point spread function in deep wide-field adaptive optics images," A\&SA 438, 757-767 (2005).

[18] Ciliegi, P., Camera, A. L., Schreiber, L., Bellazzini, M., Bertero, M., Boccacci, P., Diolaiti, E., Foppiani, I., Lombini, M., Massari, D., Montegriffo, P., and Talia, M., "Image restoration with spatially variable PSF," in [Adaptive Optics Systems IV], Marchetti, E., Close, L. M., and Véran, J.-P., eds., SPIE-Intl Soc Optical Eng (aug 2014).

[19] Denis, L., Thiébaut, É., Soulez, F., Becker, J.-M., and Mourya, R., "Fast approximations of shift-variant blur," International Journal of Computer Vision 115(3), 253-278 (2015).

[20] Nagy, J. and O'Leary, D., "Restoring images degraded by spatially variant blur," SIAM J. Sci. Comp. 19, 1063 (1998).

[21] Flicker, R. and Rigaut, F. J., "Anisoplanatic deconvolution of adaptive optics images," J Opt Soc Am A 22, 504-513 (Mar 2005).

[22] Eckart, C. and Young, G., "A principal axis transformation for non-hermitian matrices," Bulletin of the American Mathematical Society 45(2), 118-121 (1939).

[23] Mirsky, L., "Symmetric gauge functions and unitarily invariant norms," Quarterly J. Math. 11, 50-59 (1960).

[24] Eckart, C. and Young, G., "The approximation of one matrix by another of lower rank," Psychometrika 1(3), 211-218 (1936).

[25] Denis, L., Thiébaut, É., and Soulez, F., "Fast model of space-variant blurring and its application to deconvolution in astronomy," in [18th IEEE Int. Conf. Image Process.], (2011).

[26] Thévenaz, P., Blu, T., and Unser, M., "Interpolation revisited," IEEE Trans. Medical Imag. 19, 739-758 (July 2000).

[27] Hirsch, M., Sra, S., Scholkopf, B., and Harmeling, S., "Efficient filter flow for space-variant multiframe blind deconvolution," in [IEEE Comp. Vis. Pattern Recogn.], 607-614 (2010).

[28] Maalouf, E., Colicchio, B., and Dieterlen, A., "Fast deconvolution with non-invariant psf for 3-d fluorescence microscopy," in [Photonics Europe], 70001K-70001K, International Society for Optics and Photonics (2008).

[29] Mourya, R. K., Contributions to Image Restoration: From Numerical Optimization Strategies to Blind Deconvolution and Shift-variant Deblurring, PhD thesis, Université Jean Monnet, Saint-Étienne (2016).

[30] Soulez, F., Courbin, F., and Unser, M., "Back-propagating the light of field stars to probe telescope mirrors aberrations," in [SPIE Conf. on Astronomical Telescopes 8 Instrumentation], 9912, 264, SPIE Proc. (2016). 